\title{
Interação genótipo x ambiente para peso aos 365 dias em bubalinos da raça Mediterrâneo
}

\author{
Fernando Barreto Rodrigues( ${ }^{(1)}$, Paulo Luiz Souza Carneiro(2), Alcides de Amorim Ramos ${ }^{(3)}$, \\ Diego Pagung Ambrosini( ${ }^{(2)}$ e Carlos Henrique Mendes Malhado ${ }^{(2)}$
}

\begin{abstract}
(1)Instituto Federal do Norte de Minas Gerais, Fazenda Varginha, Km 02, Rodovia Salinas-Taiobeiras, CEP 39560-000 Salinas, MG, Brasil. E-mail: fernando.barreto@ifnmg.edu.br (2)Universidade Estadual Sudoeste da Bahia, Departamento de Ciências Biológicas, Avenida José Moreira Sobrinho, s/no, Jequiezinho, CEP 45206-510 Jequié, BA, Brasil. E-mail: plscarneiro@gmail.com, diegopagung@yahoo.com.br, carlosmalhado@gmail.com (3)Universidade Estadual Paulista, Departamento de Produção Animal, Distrito Rubião Júnior, s/no, CEP 18618-970 Botucatu, SP, Brasil. E-mail: aaramos@fmvz.unesp.br
\end{abstract}

Resumo - O objetivo deste trabalho foi verificar a presença da interação genótipos x ambientes (GxA) para peso aos 365 dias de idade, em bubalinos da raça Mediterrâneo, com o uso de modelos de normas de reação via regressão aleatória. O modelo animal padrão e os modelos de normas de reação foram testados, tendo-se avaliado o modelo hierárquico norma de reação (MNR) com um e com dois passos. O modelo hierárquico homocedástico de norma de reação com um passo $\left(\mathrm{MHNRHO}_{\mathrm{IP}}\right)$ apresentou o melhor ajuste, com base em três critérios de comparação. As estimativas de herdabilidade direta para esse modelo variaram de 0,19 a 0,78 , conforme a melhoria do ambiente. Além disso, houve mudança dos valores genéticos diretos (efeito escala) de alguns reprodutores, o que indica a existência de interação GxA. As correlações de Spearman foram altas, tanto na comparação do modelo animal padrão com os MNR, como na comparação entre os níveis de baixa, média e alta qualidade no $\mathrm{MHNRHO}_{1 \mathrm{p}}$. Portanto, embora haja interação GxA, a classificação dos reprodutores não é significativamente afetada pelos modelos. A maioria dos bubalinos é formada por animais de genótipo robusto, que são menos sensíveis a mudanças ambientais.

Termos para indexação: efeito escala, gradiente ambiental, modelo hierárquico, normas de reação, regressão aleatória, valor genético.

\section{Genotype $x$ environment interaction for weight at 365 days in Mediterranean buffaloes}

\begin{abstract}
The objective of this work was to verify the presence of the genotypes $\mathrm{x}$ environments interaction (GxE) for weight at 365 days of age in the Mediterranean breed of buffaloes, using models of reaction norms through random regression. The standard animal model and the models of reaction norms were tested, and the hierarchical model reaction norm (MRN) was evaluated with one and two steps. The homoscedastic hierarchical model of reaction norms with one-step $\left(\mathrm{HMRNHO}_{1 \mathrm{~s}}\right)$ had the best fit, based on three criteria for comparison. The direct heritability estimates for this model ranged from 0.19 to 0.78 , with environment improvement. Besides, direct breeding values (scale effect) changed for some breeders, which indicates the existence of GxE interaction. The Spearman correlations were high, both in the comparison between the standard animal model and MRN, as in the comparison between low, medium, and high management levels in $\mathrm{HMRNHO}_{1 \mathrm{~s}}$. Therefore, although there is GxE interaction, the ranking of breeding animals is not significantly affected by the models. The majority of buffaloes is formed by animals with robust genotypes, which are less sensitive to environmental changes.
\end{abstract}

Index terms: scale effect, environmental gradient, hierarchical model, reaction norms, random regression, breeding value.

\section{Introdução}

Os bubalinos são animais de triplo propósito, por se adaptarem à produção de leite e carne e ao trabalho (Bastianetto, 2009). Esses animais são encontrados em todos os estados brasileiros (Malhado et al.,
2007), em uma grande diversidade de ambientes e de sistemas de produção.

O Brasil foi pioneiro em avaliações genéticas de bubalinos, com a publicação do primeiro (Ramos et al., 2001) e do segundo sumário de búfalos (Ramos et al., 2004). Esses trabalhos utilizaram 
modelos mistos em suas análises estatísticas, com a consideração do modelo animal completo.

Com a expansão da bubalinocultura brasileira, diversos estudos foram publicados, principalmente sobre bubalinos leiteiros (Marcondes, 2011). Contudo, há ainda poucas informações sobre estimativas de parâmetros genéticos em búfalos de corte, especialmente no que tange à interação genótipo $\mathrm{x}$ ambiente (GxA).

A interação GxA resulta na resposta diferenciada de genótipos à variação ambiental (Falconer \& Mackay, 1996). Uma das alternativas para o estudo da interação GxA é a aplicação da norma de reação (NR), que é definida como o fenótipo expresso por um genótipo em função do ambiente (De Jong, 1995). Esses modelos são úteis nos casos em que os fenótipos mudam gradual e continuamente conforme um gradiente ambiental. Como nos modelos NR são atribuídos dois coeficientes de regressão aleatórios (intercepto e linear) a cada animal avaliado, esses coeficientes podem ser utilizados para predizer o valor genético em função do gradiente ambiental.

O objetivo deste trabalho foi verificar a interação genótipos $\mathrm{x}$ ambientes (GxA) para o peso aos 365 dias de idade, em bubalinos da raça Mediterrâneo, com o uso de modelos de normas de reação via regressão aleatória.

\section{Material e Métodos}

Utilizaram-se os registros de animais da raça Mediterrâneo, nascidos entre 1988 e 2009, pertencentes a cinco fazendas brasileiras: uma da Região Norte, outra do Nordeste, duas da Região Sul e uma do Sudeste. Os registros foram cedidos pelo Programa de Melhoramento de Bubalinos. O banco de dados inicial continha 5.280 registros de peso de animais aos 365 dias de idade (P365). Excluíramse 489 animais com registro de peso menor do que $120 \mathrm{~kg}$ e maior do que $550 \mathrm{~kg}$, além de 88 animais com registros em grupos contemporâneos (GC), com três ou menos observações e com média de peso acima de três desvios-padrão em relação à média do GC. Eliminaram-se ainda seis animais que apresentaram número de filhos menor que 2, e 142 animais que estavam em "arquipélagos secundários". Assim, utilizou-se um arquivo final com 4.555 animais, com P365 médio de 300,11 $\pm 57,04 \mathrm{~kg}$.
Inicialmente, realizaram-se análises de preparação, formatação e descrição dos dados em linguagem SAS (SAS Institute, Cary, NC, EUA). Os GC foram formados com os animais que tiveram ambiente equivalente de produção, inclusive ano, estação, rebanho e sexo. As estações de nascimento foram agrupadas em quatro classes: estação 1, setembro, outubro e novembro; estação 2, dezembro, janeiro e fevereiro; estação 3, março, abril e maio; e estação 4, junho, julho e agosto.

A conexão dos $\mathrm{GC}$, com base no número total de laços genéticos (mínimo 10), foi feita com o programa AMC (Roso \& Schenkel, 2006) e, na preparação do arquivo de pedigree e do arquivo de dados, foram incluídos somente GC conectados. A conectabilidade dos GC resultou em dois arquipélagos: o primeiro com 139 GC e 4.555 animais, e o segundo com 31 GC e 142 animais. Somente o arquipélago principal foi analisado.

Iniciou-se a análise com o modelo animal padrão (MP), com uso do programa Intergen (Cardoso, 2010), para obtenção das estimativas dos efeitos médios de ambiente, de acordo com os grupos de contemporâneos. Os modelos de normas de reação foram analisados posteriormente.

O programa Intergen ajusta modelos hierárquicos de Bayes, ou seja, modelos com parâmetros definidos - em níveis ou estágios estruturados - que contemplem a diversidade de situações comuns em dados de desempenho animal. No caso dos modelos de normas de reação, o valor genético do animal é estimado por meio de uma função do nível ambiental médio correspondente à solução do $\mathrm{GC}$ ao qual o registro pertence, isto é, para cada nível ambiental, há um valor genético específico de cada animal.

O MP ignora a interação GxA e estima o valor genético animal e o efeito ambiental posteriormente usado como covariável nos modelos de normas de reação. $\mathrm{O}$ modelo tem a seguinte expressão: $\mathrm{y}_{\mathrm{ij}}=\mathrm{x}_{\mathrm{j}}{ }^{\prime}+\mathrm{X}_{\mathrm{j}}+\mathrm{a}_{\mathrm{i}}+\varepsilon_{\mathrm{ij}}$, em que: $\mathrm{y}_{\mathrm{ij}}$ é o registro fenotípico do animal $\mathrm{i}$, no ambiente $\mathrm{j} ; \mathrm{x}_{\mathrm{j}}{ }^{\prime}$ é o vetor de incidência; $X_{j}$ é o efeito ambiental aleatório, definido para representar os efeitos de GC; $a_{i}$ é o valor genético aditivo do animal $\mathrm{i}$; e $\varepsilon_{\mathrm{ij}}$ é o erro residual.

Os modelos hierárquicos de normas de reação foram utilizados de acordo com duas metodologias: $\mathrm{MHNR}_{2 \mathrm{p}}$, modelo hierárquico norma de reação com dois passos; e $\mathrm{MHNR}_{1 \mathrm{P}}$, modelo hierárquico norma de reação com 
um passo. OMHNR 2 , proposto por Kolmodin et al. (2002), utiliza as soluções ambientais do MP como covariáveis no MHNR e tem a seguinte expressão: $\mathrm{y}_{\mathrm{ij}}=\mathrm{x}^{\prime}+\Phi \hat{\mathrm{X}}_{\mathrm{j}}+\mathrm{a}_{\mathrm{i}}+\mathrm{b}_{\mathrm{i}} \widehat{\mathrm{X}}_{\mathrm{j}}+\varepsilon_{\mathrm{ij}}$, em que: $\Phi$ é o coeficiente de regressão fixo; $a_{i}$ é o valor genético aditivo direto do intercepto ou o nível da norma de reação do animal $i ; b_{i}$ é o coeficiente de regressão aleatório ou a inclinação da norma de reação do animal $\mathrm{i}$, no ambiente $\widehat{X}_{\mathrm{j}} ; \widehat{X}_{\mathrm{j}}$ é o preditor de $\mathrm{X}_{\mathrm{j}}$ obtido no MP; e $\varepsilon_{i j}$ é o erro residual. No MHNR 1 , proposto por Su et al. (2006), as estimativas dos efeitos ambientais são obtidas juntamente com a norma de reação dos animais, e $X_{j}$ e $b_{i}$ são conjuntamente estimados, conforme a expressão $Y_{i j}=x^{\prime}+X_{j}+a_{i}+b_{i} X_{j}+\varepsilon_{i j}$.

Adotou-se a variância residual com homocedasticidade, já que a heterogeneidade de variância não convergiu nas estimações. A variância genética aditiva direta no ambiente $X\left(\sigma_{A}^{2} \mid X\right)$ foi obtida por $\sigma_{\mathrm{A}}^{2} \mid \mathrm{X}=\operatorname{var}\left(\mathrm{a}_{\mathrm{i}}+\mathrm{b}_{\mathrm{i}} \mathrm{X}_{\mathrm{j}}\right)=\sigma_{\mathrm{a}}^{2}+\sigma_{\mathrm{b}}^{2} \mathrm{X}^{2}+2 \sigma_{\mathrm{a}, \mathrm{b}} \mathrm{x}$.

As herdabilidades foram estimadas pela razão da variância genética com a variância fenotípica (genética + ambiental): $h_{\mathrm{a}}^{2}\left|\mathrm{X}=\sigma_{\mathrm{a}}^{2}\right| \mathrm{X} / \sigma_{\mathrm{a}}^{2}\left|\mathrm{X}+\sigma_{\mathrm{e}}^{2}\right| \mathrm{X}$, em que: $\sigma_{\mathrm{e}}^{2} \mid \mathrm{X}$ é a variância residual no ambiente $\mathrm{X}$, obtida por $\sigma_{\mathrm{e}}^{2}$ no modelo homocedástico, conforme Cardoso et al. (2005).

A análise de convergência das cadeias, para os diferentes modelos foi realizada por meio do diagnóstico de Geweke (1992), que se baseia num teste $\mathrm{Z}$ de igualdade de médias, do logaritmo da distribuição condicional dos dados $-1_{\mathrm{i}}^{(\mathrm{j})}=\log \mathrm{p}\left(\mathrm{y} \mid \theta^{(\mathrm{j})}, \mathrm{M}_{\mathrm{i}}\right)$ - das primeiras amostras (10\% iniciais) e da última parte da cadeia de Markov (últimas 50\%), similarmente ao proposto por Brooks \& Roberts (1998). A análise utiliza a seguinte estatística:

$$
\begin{aligned}
& \mathrm{Z}_{\mathrm{i}}=\left(\overline{\mathrm{l}}_{\mathrm{i}}^{\mathrm{A}}-\overline{\mathrm{1}}_{\mathrm{i}}^{\mathrm{B}}\right) / \sqrt{\frac{1}{\mathrm{n}_{\mathrm{A}}} \widehat{\mathrm{S}}_{\mathrm{i}}^{\mathrm{A}}(\mathrm{O})+\frac{1}{\mathrm{n}_{\mathrm{B}}} \widehat{\mathrm{S}}_{\mathrm{i}}^{\mathrm{B}}(\mathrm{O})} ; \\
& \text { em que: } \overline{1}_{\mathrm{i}}^{\mathrm{A}}=\frac{1}{\mathrm{n}_{\mathrm{A}}} \sum_{\mathrm{j}-1}^{\mathrm{n}_{\mathrm{A}}} \mathrm{l}_{\mathrm{i}}^{(\mathrm{j})} ; \overline{1}_{\mathrm{i}}^{\mathrm{B}}=\frac{1}{\mathrm{n}_{\mathrm{B}}} \sum_{\mathrm{j}-\mathrm{n}^{*}}^{\mathrm{m}} \mathrm{l}_{\mathrm{i}}^{(\mathrm{j})},
\end{aligned}
$$

em que: $\mathrm{n}_{\mathrm{A}}=120.000, \mathrm{n}_{\mathrm{B}}=600.000$ !, e $\mathrm{n}^{*}=600.001$, com $\widehat{\mathrm{S}}_{\mathrm{i}}^{\mathrm{A}}(\mathrm{O})$ e $\widehat{\mathrm{S}}_{\mathrm{i}}^{\mathrm{B}}(\mathrm{O})$, que são as respectivas estimativas da densidade espectral na frequência zero, obtida com o procedimento Spectra do SAS (SAS Institute, Cary, $\mathrm{NC}, \mathrm{EUA}$ ), para os primeiros $\mathrm{n}_{\mathrm{A}}$ e últimos $\mathrm{n}_{\mathrm{B}}$ (ciclos da cadeia MCMC de comprimento $\mathrm{m}$ ). Valores absolutos extremos do escore $\mathrm{Z}_{\mathrm{i}}$, para o teste de duas caudas, indicaram rejeição do teste de convergência.

$\mathrm{O}$ ajuste dos diferentes modelos (MP, $\mathrm{MHNRHO}_{2 \mathrm{P}}$ e $\mathrm{MHNRHO}_{1 \mathrm{P}}$ ) foi avaliado por meio de três critérios: DIC, critério de informação da "deviance"; BF, "deviance" baseada nos fatores de Bayes; e CPO, "deviance" baseada na ordenada preditiva condicional.

\section{Resultados e Discussão}

Todos os parâmetros do MP e do $\mathrm{MHNRHO}_{2 \mathrm{P}}$ convergiram a $5 \%$ de probabilidade; enquanto os parâmetros do $\mathrm{MHNRHO}_{1 \mathrm{P}}$ convergiram a $1 \%$ de probabilidade (Tabela 1).

O MHNRHO $_{1 \mathrm{P}}$ ajustou-se melhor aos dados, conforme os três critérios de comparação utilizados (Tabela 2). Resultados semelhantes foram relatados por Corrêa (2007), Cardoso et al. (2011), Mattar et al. (2011) e Ambrosini et al. (2014), que avaliaram o ganho de peso padronizado pós-desmama, para as raças Angus, Devon, Hereford, Canchim e Nelore, respectivamente. Os parâmetros e valores genéticos são estimados de forma conjunta e simultânea pelo $\mathrm{MHNRHO}_{1 \mathrm{P}}$. Isto diminui o viés, em razão da utilização prévia de informação obtida do MP, no procedimento com dois passos .

Tabela 1. Teste de convergência de Geweke para os diversos componentes dos modelos.

\begin{tabular}{lccc}
\hline Teste de Geweke & \multicolumn{3}{c}{ Modelo } \\
\cline { 2 - 4 } & Padrão & MHNRHO $_{1 \mathrm{P}}$ & $\mathrm{MHNRHO}_{2 \mathrm{P}}$ \\
\hline \multirow{3}{*}{ Parâmetro VC1 } \\
Valor Z & $-0,33^{*}$ & $0,98^{*}$ & $-1,94^{*}$ \\
Valor p & 0,74 & 0,33 & 0,06 \\
\hline \multicolumn{3}{c}{ Parâmetro VC2 } \\
Valor Z & $1,33^{*}$ & $0,04^{*}$ & $0,17^{*}$ \\
Valor p & 0,18 & 0,96 & 0,86 \\
\hline \multicolumn{3}{c}{ Parâmetro VC3 } \\
Valor Z & $0,34^{*}$ & $1,73^{*}$ & $0,37^{*}$ \\
Valor p & 0,74 & 0,08 \\
\hline \\
Valor Z & \multicolumn{3}{c}{0,70} \\
Valor p & - & $2,44^{* *}$ & $0,46^{*}$ \\
\hline & - & 0,02 & 0,64 \\
Valor Z & \multicolumn{3}{c}{ Parâmetro VC5 } \\
Valor p & - & $-0,70^{*}$ & $-0,08^{*}$ \\
\hline
\end{tabular}

$* \mathrm{e}^{* *}$ Significativo pelo teste Geweke a 5 e $1 \%$ de probabilidade, respectivamente. $\mathrm{VC} 1$, variância do efeito animal (MP); VC2, variância do grupo de contemporâneos (MP e MHNRs); VC3, correlação entre nível e inclinação da norma de reação (MHNRs); VC4, variância da inclinação da norma de reação (MHNRs); e VC5, variância do erro residual (MA e MHNRs). 
A estimativa de herdabilidade direta para o MP foi de 0,46. Malhado et al. (2008), com o uso da metodologia da máxima verossimilhança restrita (REML), relataram valores de herdabilidade direta próximos a esses $(0,41 \pm 0,06)$, para esta mesma raça, quanto ao peso aos 365 dias de idade.

As soluções do gradiente ambiental padronizadas variaram de -66 a $66 \mathrm{~kg}$, o que indica a ocorrência de ambientes de baixa à alta qualidade. As estimativas de herdabilidade direta do $\mathrm{MHNRHO}_{1 \mathrm{P}}$ aumentaram $(0,19$ a 0,78$)$ com a melhoria do gradiente ambiental (Figura 1). O aumento da proporção dos fatores genéticos em relação aos fatores ambientais da variação fenotípica com a melhoria do ambiente, bem como as mudanças nos parâmetros genéticos conforme o ambiente, também foram relatados por Kolmodin et al. (2002), Su et al. (2006) e Cardoso \& Tempelman (2012), em estudos sobre bovinos. Esses resultados indicam interação GxA, que deve ser comprovada pela avaliação da classificação dos reprodutores. Quando há mudança na classificação, considera-se que a interação é complexa e, quando os genótipos apresentam respostas distintas ao gradiente ambiental, considera-se que a interação GxA apresenta efeito escala. Independentemente do tipo de interação, os resultados obtidos evidenciam que a seleção em ambientes melhores permite melhor discriminação da variância genética aditiva.

Ao utilizar o $\mathrm{MHNRHO}_{2 \mathrm{p}}$, Corrêa et al. (2009) e Cardoso et al. (2011) também estimaram herdabilidades diretas, quanto ao ganho pós-desmama de bovinos Devon e Hereford, respectivamente, com valores próximos aos do presente estudo $(0,29$ a 0,70 e 0,07 a 0,64$)$.

Tabela 2. Avaliação dos modelos de acordo com: critério de "deviance", baseado nos fatores de Bayes (FB); critério de informação da deviance (DIC); e critério de "deviance" baseado na ordenada preditiva condicional $(\mathrm{CPO})^{(1)}$.

\begin{tabular}{lccc}
\hline Modelo & DIC & CPO & FB \\
\hline Modelo padrão & $56.703,13\left(3^{\circ}\right)$ & $48.120,35\left(3^{\circ}\right)$ & $45.864,48\left(3^{\circ}\right)$ \\
MHNRHO $_{1 \mathrm{P}}$ & $56.136,34\left(1^{\circ}\right)$ & $48.021,66\left(1^{\circ}\right)$ & $45.494,03\left(1^{\circ}\right)$ \\
MHNRHO $_{2 \mathrm{P}}$ & $56.436,16\left(2^{\circ}\right)$ & $48.054,03\left(2^{\circ}\right)$ & $45.808,16\left(2^{\circ}\right)$ \\
\hline
\end{tabular}

${ }^{(1)}$ Valores entre parênteses indicam a classificação dos modelos quanto ao ajuste. $\mathrm{MHNRHO}_{1 \mathrm{P}}$ e $M H N R H \mathrm{O}_{2 \mathrm{P}}$, modelos hierárquicos homocedásticos de norma de reação, avaliados por metodologia com um e dois passos, respectivamente.
As herdabilidades no $\mathrm{MHNRHO}_{1 \mathrm{P}}$ foram crescentes ao longo do gradiente ambiental, com valores maiores do que o MP nos ambientes médios e altos (Tabela 3).

A média a posteriori, obtida após o ajuste dos dados no $\mathrm{MHNRHO}_{1 \mathrm{P}}$ da correlação entre a inclinação e o intercepto da norma de reação, foi alta quanto ao efeito direto $(0,97 \pm 0,02)$. Houve resposta à melhoria do ambiente em animais com os maiores valores genéticos diretos, quanto ao P365 (Figura 2). Ou seja, à medida que o ambiente se tornou favorável, houve aumento da diferença entre os valores genéticos dos animais, uma vez que esses indivíduos expressaram melhor seu potencial. Estudos de normas de reação quanto ao crescimento e características de produção em bovinos,

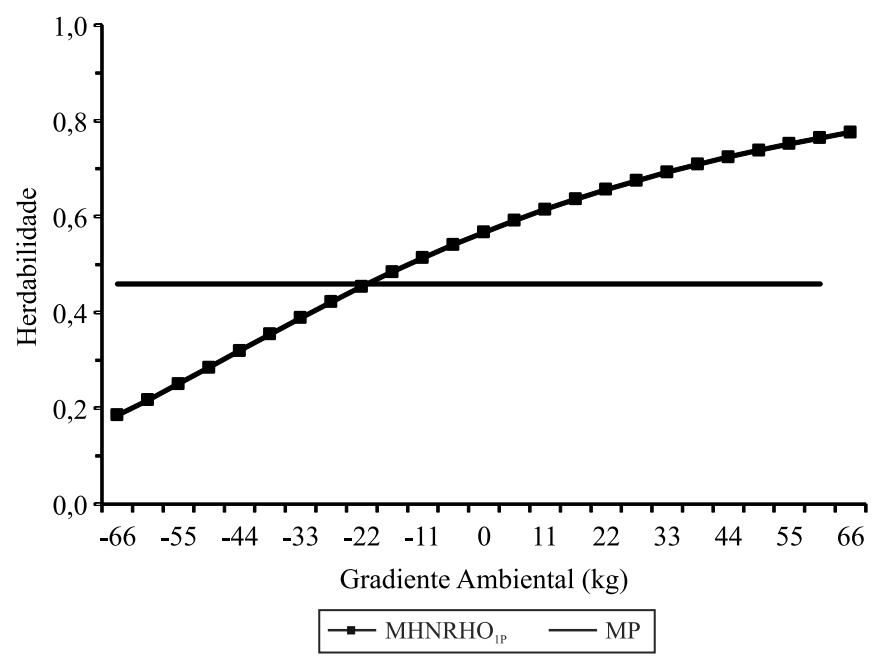

Figura 1. Herdabilidades diretas da característica P365, nos modelos avaliados, conforme o gradiente ambiental. MP, modelo animal padrão; e $\mathrm{MHNRHO}_{1 \mathrm{P}}$, modelo hierárquico homocedástico de normas de reação com um passo.

Tabela 3. Média \pm desvios-padrão da variância ambiental $\left(\sigma_{\mathrm{e}}\right)$ e da variância aditiva $\left(\sigma_{\mathrm{A}}\right)$, e herdabilidade $\left(\mathrm{h}^{2}\right)$ em diferentes níveis de ambiente $(\mathrm{X})$, considerando-se a variância residual homogênea.

\begin{tabular}{lcccc}
\hline Modelo & $\mathrm{X}(\mathrm{kg})$ & $\sigma_{\mathrm{e}}$ & $\sigma_{\mathrm{A}}$ & $\mathrm{h}^{2}$ \\
\hline Modelo padrão & - & $1.147,0 \pm 70,98$ & $975,3 \pm 104,73$ & $0,46 \pm 0,04$ \\
MHNRHO $_{1 \mathrm{P}}$ & -66 & $1.037,7 \pm 67,60$ & $236,7 \pm 74,45$ & $0,19 \pm 0,05$ \\
MHNRHO $_{1 \mathrm{P}}$ & 0 & $1.037,7 \pm 67,60$ & $1.361,5 \pm 148,30$ & $0,57 \pm 0,04$ \\
MHNRHO $_{1 \mathrm{P}}$ & +66 & $1.037,7 \pm 67,60$ & $3.596,4 \pm 472,47$ & $0,78 \pm 0,03$ \\
\hline
\end{tabular}

$\mathrm{MHNRHO}_{1 \mathrm{P}}$ e $\mathrm{MHNRHO}_{2 \mathrm{P}}$, modelos hierárquicos homocedásticos de norma de reação, avaliados por metodologia com um e dois passos, respectivamente. 
como os de Cromie (1999), Kolmodin et al. (2002), Fikse et al. (2003) e Corrêa et al. (2009), confirmam essa expectativa.

A mudança dos valores genéticos diretos (efeito escala) de alguns reprodutores, conforme o ambiente, mostra a presença de interação GxA significativa (Figura 2). As inclinações das normas de reação (efeito direto) variaram de $-1,11$ a 1,01 graus, e $59,1 \%$ dos animais (3.475) foram classificados como robustos ou intermediários (inclinações entre $-0,20$ e 0,20 graus), e $40,9 \%$ dos animais (2.405), como plásticos (-1,11 a $-0,20$ e 1,01 a 0,20$)$. Porém, apesar de a maioria dos animais ter sido classificada como robusta, a análise dos animais com maior número de filhos (animais $\mathrm{B}$ e C) permite constatar interação GxA com efeito escala (Figura 2).

As correlações de Spearman entre as classificações dos reprodutores, nos diferentes ambientes (baixo, médio e alto), pelos modelos MP e $\mathrm{MHNRHO}_{1 \mathrm{p}}$, variaram de 0,89 a 1,00, quando foram escolhidos $50 \%$ dos reprodutores com maiores valores genéticos (abaixo da diagonal). Quando se consideraram todos os reprodutores (acima da diagonal), as correlações foram de 0,91 a 0,99 (Tabela 4). Todas as correlações foram altas, tanto na comparação do MP com os MNR, como na comparação entre os níveis de baixa, média e alta qualidade do $\mathrm{MHNRHO}_{1 \mathrm{p}}$. Isto evidencia poucas mudanças na classificação dos animais, nos diferentes ambientes. Assim, não se observou mudança na

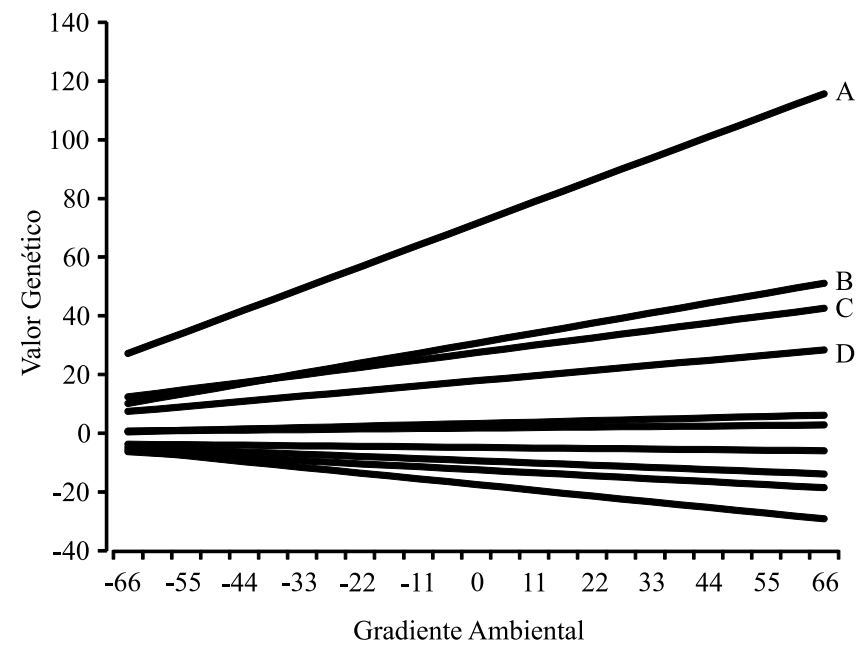

Figura 2. Norma de reação ao longo do gradiente ambiental, para a variável P365, na avaliação do efeito direto obtido para os 10 reprodutores com maior número de filhos. classificação dos reprodutores, mesmo com mudanças das variâncias genéticas aditivas ao longo do gradiente ambiental e de resposta diferenciada de alguns genótipos, avaliada pela correlação entre o intercepto e a norma de reação.

Esses resultados corroboram os Ambrosini et al. (2012), que também não observaram mudanças consideráveis na classificação dos animais, quando compararam os níveis ambientais baixo, médio e alto no $\mathrm{MHNRHO}_{1 \mathrm{p}}$. No entanto, Corrêa (2007) relatou grande alteração na classificação dos reprodutores da raça Devon, com uso de MNR.

As correlações entre os valores genéticos, nos diferentes gradientes ambientais no $\mathrm{MHNRHO}_{\mathrm{lp}}$, foram de 0,87 a 1,00, quanto ao efeito direto (Figura 3). A correlação genética foi alta e positiva, entre os valores médios e altos do gradiente ambiental, e diminuiu à medida que um dos ambientes se tornou desfavorável. Esses resultados diferem dos encontrados por Corrêa et al. (2009), que observaram correlações genéticas negativas entre os níveis do gradiente ambiental, quanto ao ganho pós-desmama, padronizado aos 365 dias, em bovinos Devon. Todavia, Mattar et al. (2011), ao avaliar peso ao sobreano em bovinos Canchim, observaram valores entre 0,24 e 1,00, com diminuição dessas correlações somente em ambientes extremamente desfavoráveis. As correlações obtidas no presente trabalho foram maiores do que as relatadas para bovinos (Corrêa et al., 2009; Mattar et al., 2011), com a evidência de menor interação GxA quanto ao P365 em bubalinos.

Tabela 4. Correlações de Spearman entre as classificações dos reprodutores com maiores valores genéticos ${ }^{(1)}$, obtidas pelo modelo padrão e pelo modelo hierárquico homocedástico de normas de reação $\left(\mathrm{MHNRHO}_{\mathrm{IP}}\right)$, quanto aos diferentes níveis ambientais ${ }^{(2)}$.

\begin{tabular}{|c|c|c|c|c|}
\hline Modelo & $\begin{array}{c}\text { Modelo } \\
\text { padrão }\end{array}$ & $\begin{array}{c}\mathrm{MHNRHO}_{1 \mathrm{P}} \\
\text { (Baixo) }\end{array}$ & $\begin{array}{l}\mathrm{MHNRHO}_{1 \mathrm{P}} \\
\text { (Médio) }\end{array}$ & $\begin{array}{l}\mathrm{MHNRHO}_{1 \mathrm{P}} \\
\text { (Alto) }\end{array}$ \\
\hline Modelo padrão & - & $\begin{array}{c}0,91 \\
(<0,001)\end{array}$ & $\begin{array}{c}0,93 \\
(<0,001)\end{array}$ & $\begin{array}{c}0,93 \\
(<0,001)\end{array}$ \\
\hline $\begin{array}{l}\text { MHNRHO }_{1 \mathrm{P}} \\
\text { (Baixo) }\end{array}$ & $\begin{array}{c}0,91 \\
(<, 0001)\end{array}$ & - & $\begin{array}{c}0,99 \\
(<0,001)\end{array}$ & $\begin{array}{c}0,99 \\
(<0,001)\end{array}$ \\
\hline $\begin{array}{l}\text { MHNRHO }_{1 \mathrm{P}} \\
\text { (Médio) }\end{array}$ & $\begin{array}{c}0,90 \\
(<, 0001)\end{array}$ & $\begin{array}{c}0,99 \\
(<0,001)\end{array}$ & - & $\begin{array}{c}0,99 \\
(<0,001)\end{array}$ \\
\hline $\begin{array}{l}\text { MHNRHO }_{1 \mathrm{P}} \\
\text { (Alto) }\end{array}$ & $\begin{array}{c}0,89 \\
(<, 0001) \\
\end{array}$ & $\begin{array}{c}0,98 \\
(<0,001)\end{array}$ & $\begin{array}{c}1,00 \\
(<0,001)\end{array}$ & 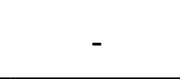 \\
\hline
\end{tabular}

${ }^{(1)} \mathrm{A}$ avaliação considerou $50 \%$ de reprodutores com os maiores valores genéticos (abaixo da diagonal) ou 100\% dos animais (acima da diagonal).

${ }^{(2)}$ Valores entre parênteses indicam a significância das correlações. 


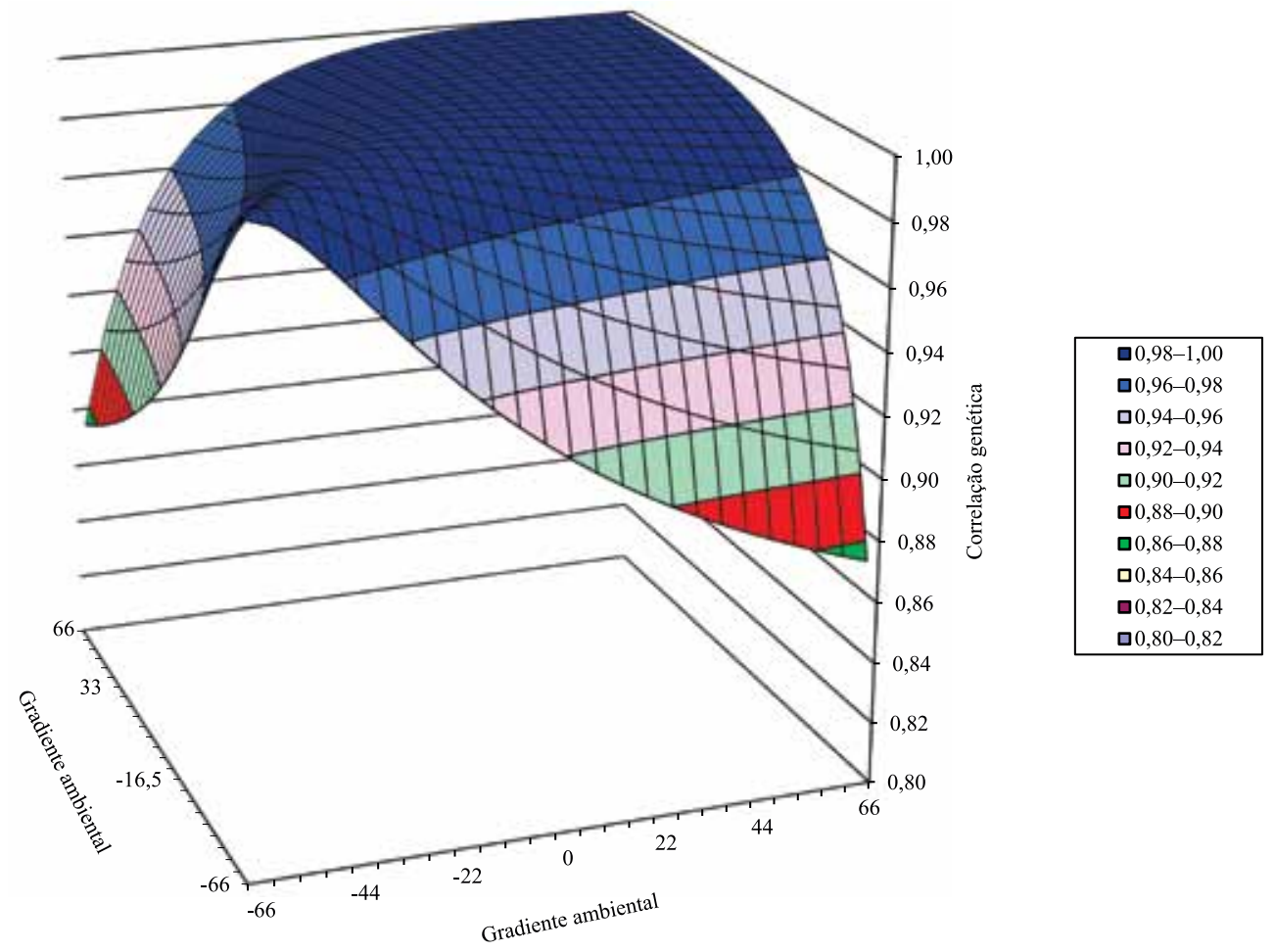

Figura 3. Superfície de resposta das correlações entre os valores genéticos diretos e o gradiente ambiental.

\section{Conclusões}

1. O modelo de norma de reação com um passo $\left(\mathrm{MHNRHO}_{1 \mathrm{P}}\right)$ é o que apresenta melhor ajuste aos dados, com aumento das herdabilidades ao longo do gradiente ambiental.

2. A interação genótipo $\mathrm{x}$ ambiente, para os reprodutores com maior número de filhos, apresenta efeito escala; entretanto, a classificação dos animais não se altera significativamente com o uso do modelo animal padrão ou do $\mathrm{MHNRHO}_{1 \mathrm{P}}$, e nem na comparação entre os níveis ambientais.

2. A maioria dos bubalinos é formada por animais de genótipo robusto, que são menos sensíveis a mudanças ambientais.

\section{Agradecimentos}

Ao Conselho Nacional de Desenvolvimento Científico e Tecnológico (CNPq, processo 302378/2012-0), pela concessão de bolsa produtividade.

\section{Referências}

AMBROSINI, D.P.; MALHADO, C.H.M.; BRACCINI NETO, J.; MARTINS FILHO, R.; AFFONSO, P.R.A. de M.; CARNEIRO, P.L.S. Reaction norms of direct and maternal effects for weight at 205 days in Polled Nellore cattle in North-eastern Brazil. Archiv Tierzucht, v.57, article 32, 2014. DOI: 10.7482/0003-9438-57-032.

AMBROSINI, D.P.; CARNEIRO, P.L.S.; BRACCINI NETO, J.; MALHADO, C.H.M.; MARTINS FILHO, R.; CARDOSO, F.F. Interação genótipo $\mathrm{x}$ ambiente para peso ao ano em bovinos Nelore Mocho no Nordeste do Brasil. Pesquisa Agropecuária Brasileira, v.47, p.1489-1495, 2012. DOI: 10.1590/ S0100-204X2012001000011.

BASTIANETTO, E. Criação de búfalos no Brasil: situação e perspectiva. Revista Brasileira de Reprodução Animal, n.6, p.98-103, 2009.

BROOKS, S.P.; ROBERTS, G.O. Convergence assessment techniques for Markov chain Monte Carlo. Statistics and Computing, v.8, p.319-335, 1998. DOI: 10.1023/A:1008820505350.

CARDOSO, F.F.; TEMPELMAN, R.J. Linear reaction norm models for genetic merit prediction of Angus cattle under genotype by environment interaction. Journal of Animal Science, v.90, p.2130-2141, 2012. DOI: 10.2527/jas.2011-4333. 
CARDOSO, L.L.; BRACCINI NETO, J.; CARDOSO, F.F.; COBUCI, J.A.; BIASSUS, I. de O.; BARCELLOS, J.O.J. Hierarchical Bayesian models for genotype $\mathrm{x}$ environment estimates in post-weaning gain of Hereford bovine via reaction norms. Revista Brasileira de Zootecnia, v.40, p.294-300, 2011. DOI: $10.1590 / \mathrm{S} 1516-35982011000200009$.

CARDOSO, F.F. Aplicação da inferência bayesiana no melhoramento animal usando o programa Intergen: manual da versão 1.2. Bagé: Embrapa Pecuária Sul, 2010. 31p. (Embrapa Pecuária Sul. Documentos, 111).

CARDOSO, F.F.; ROSA, G.J.M.; TEMPELMAN, R.J. Multiple-breed genetic inference using heavy-tailed structural models for heterogeneous residual variances. Journal of Animal Science, v.83, p.1766-1779, 2005.

CORRÊA, M.B.B. Caracterização da interação genótipo-ambiente no desempenho de bovinos Devon no Rio Grande do Sul via normas de reação obtidas por regressão aleatória. 2007. 88p. Tese (Doutorado) - Universidade Federal de Pelotas, Pelotas.

CORRÊA, M.B.B.; DIONELlO, N.J.L.; CARDOSO, F.F. Caracterização da interação genótipo-ambiente e comparação entre modelos para ajuste do ganho pós-desmama de bovinos Devon via normas de reação. Revista Brasileira de Zootecnia, v.38, p.1468-1477, 2009. DOI: 10.1590/ S1516-35982009000800010.

CROMIE, A.R. Genotype by environment interaction for milk production traits in Holstein Friesian dairy cattle in Ireland. 1999. Thesis (PhD) - Queen's University of Belfast, Belfast.

DE JONG, G. Phenotypic plasticity as a product of selection in a variable environment. The American Naturalist, v.145, p.493-512. 1995. DOI: 10.1086/285752.

FALCONER, D.S.; MACKAY, T.F.C. Introduction to quantitative genetics. $4^{\text {th }}$ ed. Edinburgh: Pearson, 1996. 464p.

FIKSE, W.F.; REKAYA, R.; WEIGEL, K.A. Assessment of environmental descriptors for studying genotype by environment interaction. Livestock Production Science, v.82, p.223-231, 2003. DOI: 10.1016/S0301-6226(03)00009-5.

GEWEKE, J. Evaluating the accuracy of sampling-based approaches to the calculation of posterior moments. In: BERNARDO, J.M.; BERGER, J.O.; DAWID, A.P.; SMIT,
A.F.M. (Ed.). Bayesian statistics 4. Oxford: Oxford University, 1992. 526p.

KOLMODIN, R.; STRANDBERG, E.; MADSEN, P.; JENSEN, J.; JORJANI, H. Genotype by environment interaction in Nordic dairy cattle studied using reaction norms. Acta Agriculturae Scandinavia, Section A - Animal Science, v.52, p.11-24, 2002. DOI: $10.1080 / 09064700252806380$.

MALHADO, C.H.M.; RAMOS, A. de A.; CARNEIRO, P.L.S.; SOUZA, J.C. de; PICCININ, A. Parâmetros e tendências da produção de leite em bubalinos da raça Murrah no Brasil. Revista Brasileira de Zootecnia, v.36, p.376-379, 2007. DOI: 10.1590/ S1516-35982007000200014.

MALHADO, C.H.M.; RAMOS, A. de A.; CARNEIRO, P.L.S.; AZEVEDO, D.M.M.R.; MARTINS FILHO, R.; SOUZA, J.C. de. Melhoramento e estrutura populacional em bubalinos da raça Mediterrâneo no Brasil. Pesquisa Agropecuária Brasileira, v.43, p.215-220, 2008. DOI: 10.1590/S0100-204X2008000200009.

MARCONDES, C.R. Melhoramento de búfalos no Brasil: avanços, entraves e perspectivas. Revista Brasileira de Zootecnia, v.40, p.307-315, 2011.

MATTAR, M.; SILVA, L.O.C.; ALENCAR, M.M.; CARDOSO, F.F. Genotype x environment interaction for long-yearling weight in Canchim cattle quantified by reaction norm analysis. Journal of Animal Science, v.89, p.2349-2355, 2011. DOI: 10.2527/ jas.2010-3770.

RAMOS, A.A. (Coord.). PROMEBUL: sumário de touros bubalinos. Botucatu: Unesp, 2001. 22p. (PROMEBUL. Boletim técnico, 1).

RAMOS, A.A.; WECHSLER, F.S.; VAN ONSELEN, V.J.; GONÇALVES, H.C. PROMEBUL: sumário de touros bubalinos. Botucatu: Unesp, 2004. 23p. (PROMEBUL. Boletim técnico, 2).

ROSO, V.M.; SCHENKEL, F.S. AMC - a computer program to assess the degree of connectedness among contemporary groups. In: WORLD CONGRESS ON GENETICS APPLIED TO LIVESTOCK PRODUCTION, 8., 2006, Belo Horizonte. Proceedings. Belo Horizonte: [s.n.], 2006. p.26-27.

SU, G.; MADSEN, P.; LUND, M.S.; SORENSEN, D.; KORSGAARD, I.R.; JENSEN, J. Bayesian analysis of the linear reaction norm model with unknown covariates. Journal of Animal Science, v.84, p.1651-1657, 2006. DOI: 10.2527/jas.2005-517.

Recebido em 18 de novembro de 2014 e aprovado em 27 de maio de 2015 